\title{
Performance and sex difference in ultra-triathlon performance from Ironman to Double Deca Iron ultra-triathlon between 1978 and 2013
}

\author{
Christoph A Rüst ${ }^{1}$, Thomas Rosemann ${ }^{1}$ and Beat Knechtle $e^{2^{*}}$
}

\begin{abstract}
It was assumed that women would be able to outperform men in ultra-marathon running. The present study investigated the sex difference in performance for all ultra-triathlon distances from the Ironman distance (i.e. $3.8 \mathrm{~km}$ swimming, $180 \mathrm{~km}$ cycling and $42 \mathrm{~km}$ running) in the 'Ironman Hawaii' to the Double Deca Iron ultra-triathlon distance (i.e. $76 \mathrm{~km}$ swimming, 3,600 km cycling and $840 \mathrm{~km}$ running) between 1978 and 2013. The changes in performance and in the sex difference in performance for the annual three fastest finishers were analysed using linear, non-linear and multi-variate regression analyses from 46,123 athletes (i.e. 9,802 women and 46,123 men). Women accounted for $11.9 \pm 5.8 \%$ of the total field and their percentage was highest in 'Ironman Hawaii' (22.1\%) and lowest in Deca Iron ultra-triathlon (6.5\%). In 'Ironman Hawaii', the sex difference decreased non-linearly in swimming, cycling, running and overall race time. In Double Iron ultra-triathlon, the sex difference increased non-linearly in overall race time. In Triple Iron ultra-triathlon, the sex difference increased non-linearly in cycling and overall race time but linearly in running. For the three fastest finishers ever, the sex difference in performance showed no change with increasing race distance with the exception for the swimming split where the sex difference increased with increasing race distance $\left(r^{2}=0.93, P=0.001\right)$. The sex differences for the three fastest finishers ever for swimming, cycling, running and overall race times for all distances from Ironman to Deca Iron ultra-triathlon were $27.0 \pm 17.8 \%, 24.3 \pm 9.9 \%, 24.5 \pm 11.0 \%$, and $24.0 \pm 6.7 \%$, respectively. To summarize, these findings showed that women reduced the sex difference in the shorter ultra-triathlon distances (i.e. Ironman distance) but extended the sex difference in longer distances (i.e. Double and Triple Iron ultra-triathlon). It seems very unlikely that women will ever outperform men in ultra-triathlons from Ironman to Double Iron ultra-triathlon.
\end{abstract}

Keywords: Woman; Man; Swimming; Cycling; Running

\section{Background}

A few years ago, Beneke et al. (2005) argued that women would be able to outperform men in ultra-marathon running. They reported that men were dominating the $216 \mathrm{~km}$ 'Badwater' ultra-marathon held at temperatures up to $55^{\circ} \mathrm{C}$ during the 1980 s and 1990s. However, in 2002 and 2003, a woman was able to outpace the fastest man by $\sim 4.5 \mathrm{~h}$ and $\sim 0.5 \mathrm{~h}$, respectively (www.badwater.com). Furthermore, since 2002, up to three women finished within the top five overall, although more men than women competed in the 'Badwater' (da Fonseca-Engelhardt et al. 2013). A similar finding of a female runner exceeding men's

\footnotetext{
* Correspondence: beat.knechtle@hispeed.ch

${ }^{2}$ Gesundheitszentrum St. Gallen, Vadianstrasse 26, St. Gallen 9001, Switzerland Full list of author information is available at the end of the article
}

ultra-marathon performance has been reported for a 45-years old woman winning the 'Deutschlandlauf', a multi-stage ultra-marathon covering a total distance of $1,200 \mathrm{~km}$ from the North to the South of Germany to be completed within 17 days (Knechtle et al. 2008). The woman finished the race in an overall race time of $124 \mathrm{~h}$ $40 \mathrm{~min}$, whereas the first man finished about $8 \mathrm{~h}$ behind her in $132 \mathrm{~h} 44 \mathrm{~min}$.

The assumption that women might outrun men in ultra-marathon running was supported by the comparison of female and male performance in different running distances. Hoffman (2008) showed that women and men who were matched for $50 \mathrm{~km}$ trail running performance also similarly performed in trail runs of $80 \mathrm{~km}$ and $161 \mathrm{~km}$ race distances. Physiological assumptions regarding why 
women could outrun men were differences in the ability to run aerobically at a higher percentage of maximal oxygen uptake (Riddell et al. 2003), differences in the use of ingested and stored glycogen (Riddell et al. 2003) and differences in lipid metabolism (Perreault et al. 2004). During long-duration exercise at a moderate intensity, women showed a higher lipid utilization and a lower carbohydrate and protein metabolism compared to equally trained and nourished men (Tarnopolsky et al. 1990). In addition, women used more fat stored in the legs compared to men (Roepstorff et al. 2002). Coast et al. (2004) compared by the end of 2002 the world best running performances from $100 \mathrm{~m}$ to $200 \mathrm{~km}$. Men were on average $\sim 12.4 \%$ faster than women. There was a significant slope to the speed difference across distances where longer distances were associated with greater sex differences (Coast et al. 2004). Therefore, the distance for a woman to outperform a man should be most probably longer than $200 \mathrm{~km}$ as it has been found in the $216 \mathrm{~km}$ 'Badwater' and the $1,200 \mathrm{~km}$ 'Deutschlandlauf'. However, other reasons such as participation trends and motivational trends might limit women's ultra-endurance performance. Generally, men are over-represented in sports (Deaner 2013) and the better male performance is most probably due to men's greater training motivation (Deaner 2013). In contrast, women in ultra-endurance are rather task-oriented and internally motivated (Krouse et al. 2011).

In ultra-endurance, long-distance triathlon is an interesting model investigating the sex difference in ultra-endurance because the sex difference in performance can be examined for each split discipline such as swimming, cycling and running and for the overall race time (Lepers and Maffiuletti 2011). Since the first Ironman distance triathlon held in Hawaii in 1978 consisting in $3.8 \mathrm{~km}$ swimming, $180 \mathrm{~km}$ cycling and $42.195 \mathrm{~km}$ running (Lepers 2008), the ultra-distance triathlon has been expanded to the Double Deca Iron ultra-triathlon distance consisting in $76 \mathrm{~km}$ swimming, 3,600 km cycling, and $840 \mathrm{~km}$ running (Lenherr et al. 2012).

Regarding the performance in the split disciplines in triathlon, recent studies showed that women were able to achieve a similar performance as men in ultra-distance swimming (Eichenberger et al. 2012a; 2012b). Indeed, a very recent study showed that women were able to outperform men in open-water ultra-distance swimming (Knechtle et al. 2014). In the $46 \mathrm{~km}$ 'Manhattan Island Marathon Swim' with water temperatures $<20^{\circ} \mathrm{C}$, the top ten race times ever were significantly lower for women than for men. A potential explanation for the sex difference in ultra-endurance swimming performance could be anthropometric differences such as body fat between female and male ultra-endurance athletes. Female ultra-swimmers have a higher percent body fat of $30.7 \pm 3.7 \%$ compared to male ultra-swimmers with $18.8 \pm 4.5 \%$ (Weitkunat et al. 2012). Additionally, female swimmers had proportionately more fatty tissue caudally than male swimmers (McLean and Hinrichs 1998). These findings might explain why women were able to achieve men's performance in ultra-swimming due to an improved buoyancy.

Regarding cycling as the second discipline in triathlon, little is known about the sex difference in ultra-cycling (Rüst et al. 2013; Shoak et al. 2013; Zingg et al. 2013). For shorter cycling distances, Schumacher et al. (2001) reported a sex difference of $\sim 11 \%$ in all disciplines and at all ages when investigating results of the world track cycling championships in $200 \mathrm{~m}, 1,000 \mathrm{~m}$, individual and team pursuit races for elite and junior athletes. For longer distances, women reduced the gender gap in the $720 \mathrm{~km}$ 'Swiss Cycling Marathon', the largest European qualifier for the 'Race Across America' (Zingg et al. 2013). In the 'Race Across America' itself, the sex difference remained, however, unchanged at $~ 19 \%$ for the annual fastest and at $\sim 25 \%$ for the annual three fastest in the last 30 years (Rüst et al. 2013).

Analysing the sex difference in performance in longdistance triathlons could give some additional insights regarding the sex difference in ultra-swimming, ultracycling and ultra-running performances. The aim of this study was therefore to investigate the sex difference in ultra-triathlon performance for each split discipline and overall race time in different triathlon distances ranging from the Ironman distance (i.e. $3.8 \mathrm{~km}$ swimming, $180 \mathrm{~km}$ cycling and $42 \mathrm{~km}$ running) in 'Ironman Hawaii' to the

Table 1 Number of female and male finishers in all races

\begin{tabular}{|c|c|c|c|c|c|}
\hline Race & Female finishers & Male finishers & Overall finishers & Women (\%) & Men (\%) \\
\hline Ironman Hawaii (1978-2013) & 9,502 & 33,459 & 42,961 & 22.1 & 77.9 \\
\hline Double Iron ultra-triathlon (1985-2013) & 179 & 1,731 & 1,910 & 10.3 & 89.7 \\
\hline Triple Iron ultra-triathlon (1988-2013) & 98 & 918 & 1,016 & 10.7 & 89.3 \\
\hline Quadruple Iron ultra-triathlon (1989-2013) & 3 & 39 & 42 & 7.1 & 92.9 \\
\hline Quintuple Iron ultra-triathlon (1991-2013) & 13 & 59 & 72 & 18.0 & 82.0 \\
\hline Deca Iron ultra-triathlon (1992-2013) & 8 & 123 & 128 & 6.5 & 93.5 \\
\hline Double Deca Iron ultra-triathlon (1998-2013) & 1 & 10 & 11 & 9.1 & 90.1 \\
\hline
\end{tabular}




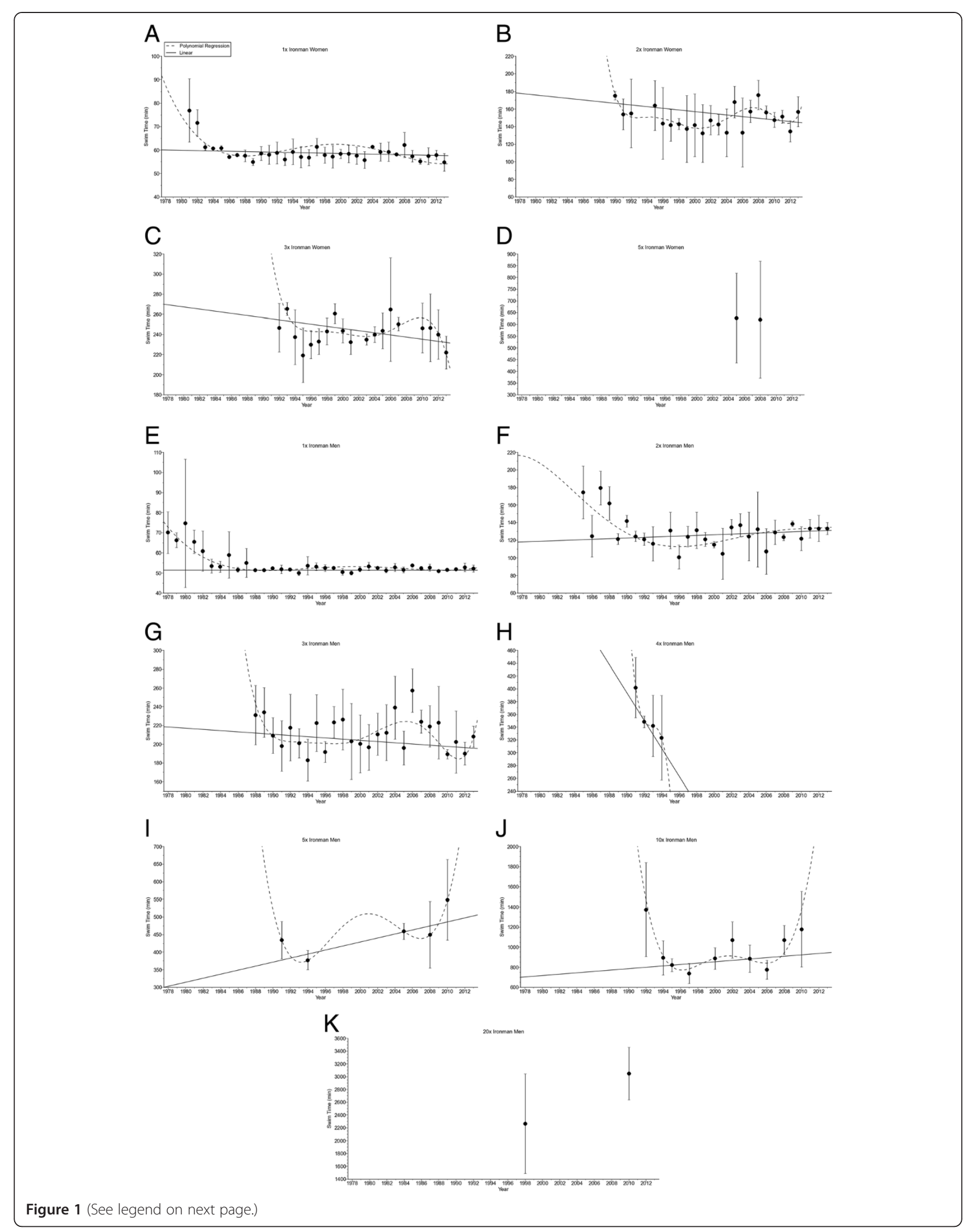


(See figure on previous page.)

Figure 1 Swimming split times for the annual three fastest women and men from Ironman to Double Deca Iron ultra-triathlon for Ironman women (Panel A), Double Iron women (Panel B), Triple Iron women (Panel C), Quintuple Iron women (Panel D), Ironman men (Panel E), Double Iron men (Panel F), Triple Iron men (Panel G), Quadruple Iron men (Panel H), Quintuple Iron men (Panel I), Deca Iron men (Panel J), Double Deca Iron men (Panel K).

Double Deca Iron ultra-triathlon distance (i.e. $76 \mathrm{~km}$ swimming, 3,600 km cycling and $840 \mathrm{~km}$ running) between 1978 and 2013. Based upon recent findings for single disciplines, we hypothesized that women would be able to reduce the gap with men in the swimming and running parts of ultra-triathlons.

\section{Methods}

\section{Ethics}

All procedures used in the study were approved by the Institutional Review Board of Kanton St. Gallen, Switzerland with a waiver of the requirement for informed consent of the participants given the fact that the study involved the analysis of publicly available data.

\section{Data sampling and data analysis}

The data for this study was obtained from the race website of 'Ironman World Championship' (www.ironmanworldchampionship.com) for race results from 'Ironman Hawaii'. For the triathlon distances between Double Iron and Double Deca Iron ultra-triathlon, the authors collected all race results from the website of the International Ultra-Triathlon Association (IUTA), www.iutasport.com) and from the race directors for all data not recorded on the website. Since the Double Iron ultra-triathlon held in Huntsville in 1994 included a downstream swimming split leading to unrealistic fast swimming split times, this specific race had to be excluded from analysis. Split times in swimming, cycling and running and overall race times of all finishers from 'Ironman Hawaii' and all ultra-triathlons up to Double Deca Iron ultra-triathlon were analysed. Due to the low number of female finishers in the longer ultra-triathlon distances (i.e. longer than the Triple Iron ultra-triathlon distance), we restricted our analysis to the three fastest triathletes ever and the annual three fastest. To find sex differences in the performance of men and women depending on the race distance, the top three men and women ever (i.e. the athletes with the three fastest overall race times ever) were sorted by race distance. From these athletes, the sex difference in performance was calculated using the equation ([time in women (min)]-[time in men ( $\mathrm{min})] /[$ time in men $(\min )] \times 100)$, where the sex difference was calculated for every pair of equally placed athletes (i.e. between male and female winner, between male and female 2nd place, etc.) before calculating mean value and standard deviation of all the pairings. In order to facilitate reading all sex differences were transformed to absolute values before analysing. The sex difference between the annual top three men and women (i.e. annual three fastest overall race times) were determined and analysed regarding the change in sex difference over time.

\section{Statistical analysis}

Each data set was tested for normal distribution and for homogeneity of variances prior to statistical analyses. Normal distribution was tested using a D'Agostino and Pearson omnibus normality test and homogeneity of variances was tested using a Levene's test. Subsequent statistical tests were corrected according to these results if indicated and necessary. Single and multi-level regression analyses were used to investigate changes in speed and sex difference. A hierarchical regression model was used to avoid the impact of a cluster-effect on results in case one athlete finished more than once in the annual top three. Since the change in sex difference in endurance is assumed to be non-linear (Reinboud 2004), we calculated both the linear and the non-linear regression model for performance and sex difference in swimming, cycling, running and overall race time. In total, over 90 different models were tested in order to find the model that fits the data the best. Among these models, polynomial regression from degree 2 to 20, exponential models, growth models, sigmoidal models, decline models and others were included. In order to find the best model explaining the

Table 2 Multi-level regression analyses for changes in swimming split times across years with correction for multiple finishes for the annual three fastest women and men

\begin{tabular}{lcccccc}
\hline Distance & Sex & $\boldsymbol{B}$ & $\mathbf{S E}(\boldsymbol{B})$ & Stand. $\boldsymbol{\beta}$ & $\mathbf{T}$ & $\boldsymbol{P}$ \\
\hline Ironman & Female & -0.203 & 0.054 & -0.358 & -3.777 & $<0.001$ \\
Double Iron & Female & -0.215 & 0.429 & -0.062 & -0.500 & 0.619 \\
Triple Iron & Female & -0.042 & 0.442 & -0.013 & -0.096 & 0.924 \\
Quintuple Iron & Female & -2.222 & 60.349 & -0.018 & -0.037 & 0.972 \\
Ironman & Male & -0.338 & 0.065 & -0.451 & -5.196 & $<0.001$ \\
Double Iron & Male & -0.525 & 0.321 & -0.175 & -1.635 & 0.106 \\
Triple Iron & Male & -0.180 & 0.417 & -0.049 & -0.431 & 0.667 \\
Quadruple Iron & Male & -24.133 & 11.354 & -0.558 & -2.126 & 0.059 \\
Quintuple Iron & Male & 5.382 & 2.526 & 0.509 & 2.131 & 0.053 \\
Deca Iron & Male & 0.727 & 8.419 & 0.016 & 0.086 & 0.932 \\
\hline
\end{tabular}


Rüst et al. SpringerPlus 2014, 3:219

Page 5 of 17

http://www.springerplus.com/content/3/1/219

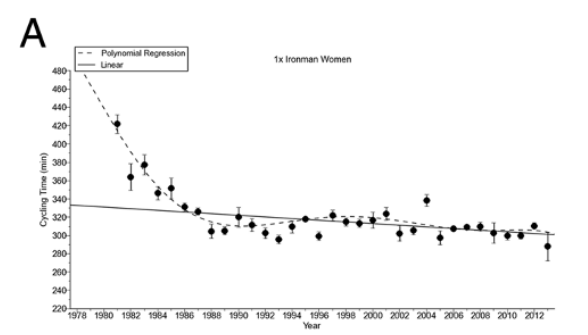

B
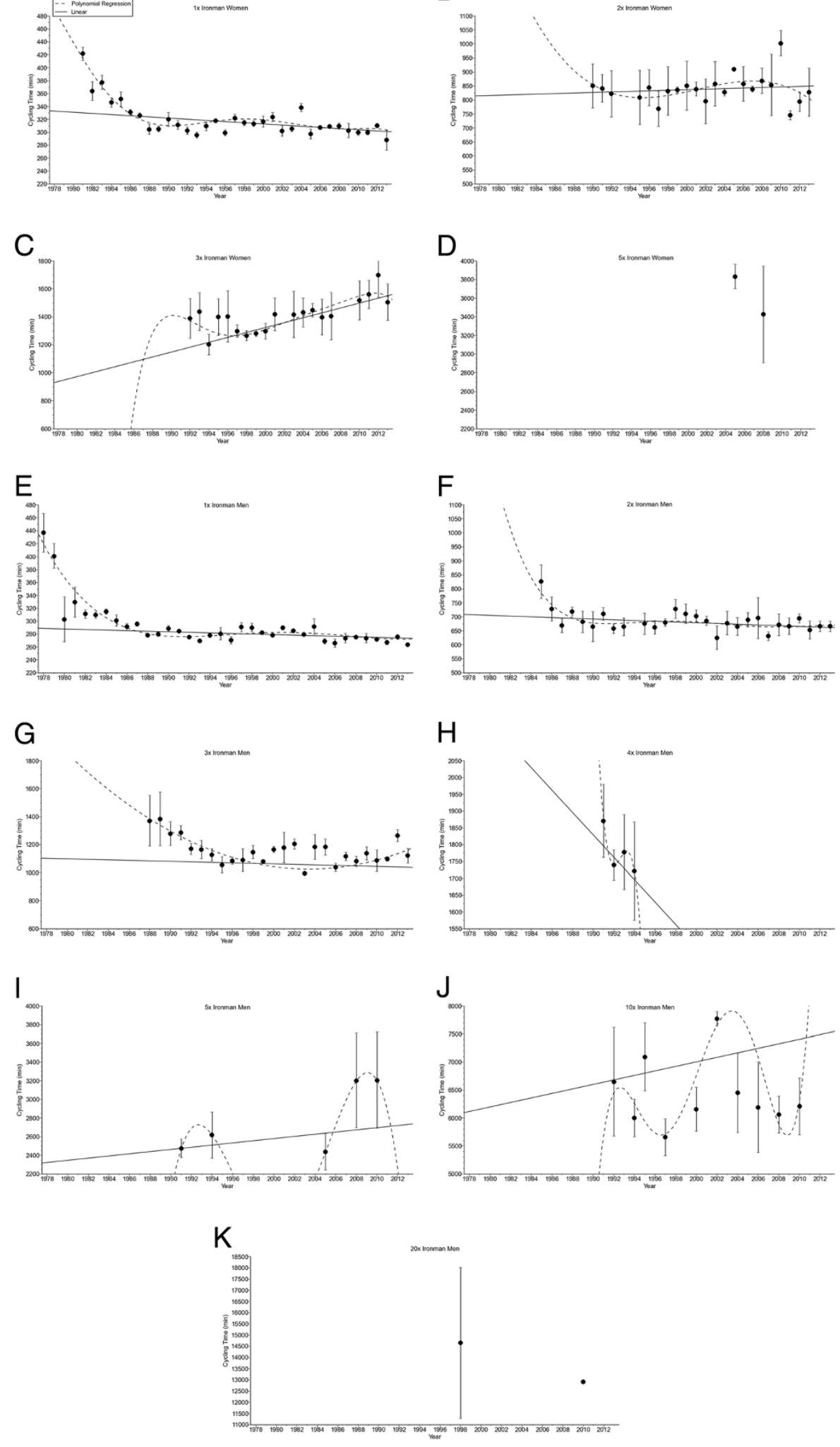

Figure 2 (See legend on next page.) 
(See figure on previous page.)

Figure 2 Cycling split times for the annual three fastest women and men from Ironman to Double Deca Iron ultra-triathlon for Ironman women (Panel A), Double Iron women (Panel B), Triple Iron women (Panel C), Quintuple Iron women (Panel D), Ironman men (Panel E), Double Iron men (Panel F), Triple Iron men (Panel G), Quadruple Iron men (Panel H), Quintuple Iron men (Panel I), Deca Iron men (Panel J), Double Deca Iron men (Panel K).

trend of the data, we compared the best-fit non-linear models to the linear model using AIC (Akaike's Information Criteria) and F-test. Since the tested sets of data showed a normal distribution as well as homogeneity of variances, the performance between two groups (e.g. men versus women), was compared using a Student's $t$-test. Additionally, a potential relationship between the sex difference of the three fastest competitors and total race time in men was investigated using Pearson's correlation analysis. Statistical analyses were performed using IBM SPSS Statistics (Version 22, IBM SPSS, Chicago, IL, USA) and GraphPad Prism (Version 6.01, GraphPad Software, La Jolla, CA, USA). Significance was accepted at $P<0.05$ (two-sided for $t$-tests). Data in the text and figures are given as mean \pm standard deviation (SD).

\section{Results}

\section{Participation trends}

Between 1978 and 2013, a total of 46,123 athletes (i.e. 9,802 women and 46,123 men) finished 'Ironman Hawaii' and ultra-triathlons longer than the 'Ironman Hawaii' (Table 1). Women accounted on average for $11.9 \pm 5.8 \%$ of the total field (Table 1 ). The percentage of female participation was highest in 'Ironman Hawaii' and lowest in Deca Iron ultra-triathlon (Table 1).

\section{Changes in performance across years}

In 'Ironman Hawaii', swimming split times (Figure 1) decreased non-linearly (i.e. polynomial regression 4th degree) (Table 2) in both women and men. From Double
Iron to Deca Iron ultra-triathlon, no changes in swimming split times were found (Table 2). Cycling split times (Figure 2) decreased non-linearly in male (i.e. polynomial regression 4th degree) and female (i.e. polynomial regression 13th degree) Ironman triathletes (Table 3), and in male Double (i.e. polynomial regression 4th degree) and male Triple Iron ultra-triathletes (i.e. polynomial regression 2nd degree) (Table 3). In Quintuple Iron ultra-triathlon, however, cycling split times increased non-linearly in men (i.e. polynomial regression 4th degree) (Table 3). No changes were found for the other distances (Table 3). Running split times (Figure 3) decreased nonlinearly in male (i.e. polynomial regression 8th degree) and female (i.e. polynomial regression 5 th degree) Ironman triathletes (Table 4). In distances longer than the Ironman, running split times decreased non-linearly in male Double Iron ultra-triathletes (i.e. polynomial regression 4th degree) (Table 4). In Triple Iron ultra-triathlon, running split times increased non-linearly in women (i.e. polynomial regression 5th degree) (Table 4). In Quintuple Iron ultra-triathlon, running split times increased nonlinearly in men (i.e. polynomial regression 4th degree) (Table 4). Overall race times (Figure 4) decreased nonlinearly in male (i.e. polynomial regression 18th degree) and female (i.e. polynomial regression 4th degree) Ironman triathletes (Table 5). In distances longer than the Ironman, overall race times decreased non-linearly in male (i.e. polynomial regression 5th degree) Double and male (i.e. polynomial regression 5 th degree) Triple Iron ultra-triathletes (Table 5). In Triple and Quintuple Iron

Table 3 Multi-level regression analyses for changes in cycling split times across years with correction for multiple finishes for the annual three fastest women and men

\begin{tabular}{|c|c|c|c|c|c|c|}
\hline Distance & Sex & $B$ & SE (ß) & Stand. B & $T$ & $P$ \\
\hline Ironman & Female & -1.816 & 0.226 & -0.632 & -8.026 & $<0.001$ \\
\hline Double Iron & Female & 0.985 & 1.320 & 0.093 & 0.746 & 0.458 \\
\hline Triple Iron & Female & 12.086 & 2.604 & 0.530 & 4.641 & $<0.001$ \\
\hline Quintuple Iron & Female & -135.778 & 102.897 & -0.551 & -1.320 & 0.257 \\
\hline Ironman & Male & -2.167 & 0.255 & -0.636 & -8.495 & $<0.001$ \\
\hline Double Iron & Male & -1.960 & 0.560 & -0.355 & -3.497 & 0.001 \\
\hline Triple Iron & Male & -6.017 & 1.563 & -0.404 & -3.849 & $<0.001$ \\
\hline Quadruple Iron & Male & -40.933 & 27.098 & -0.431 & -1.511 & 0.162 \\
\hline Quintuple Iron & Male & 32.680 & 13.618 & 0.554 & 2.400 & 0.032 \\
\hline Deca Iron & Male & -11.336 & 23.739 & -0.090 & -0.478 & 0.637 \\
\hline
\end{tabular}




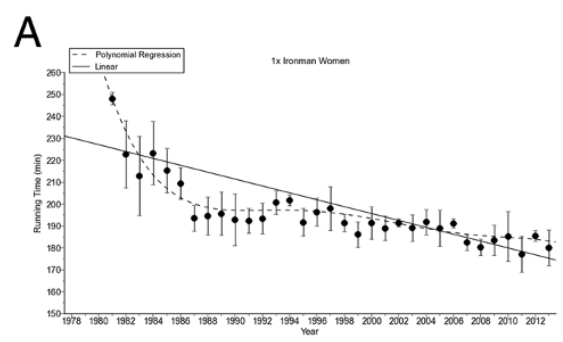

C

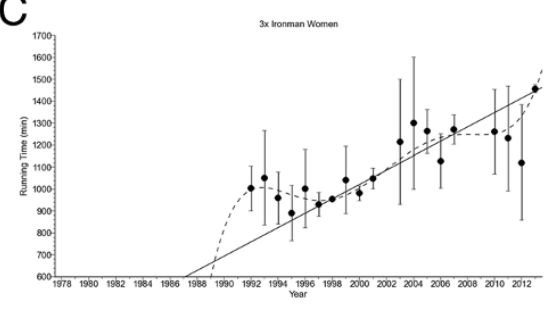

E

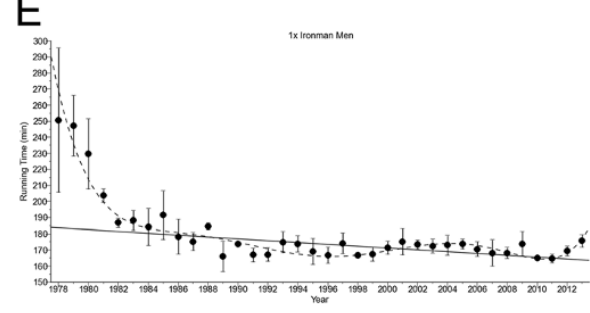

G

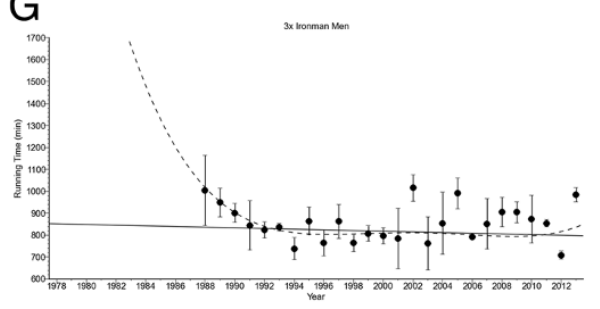

I

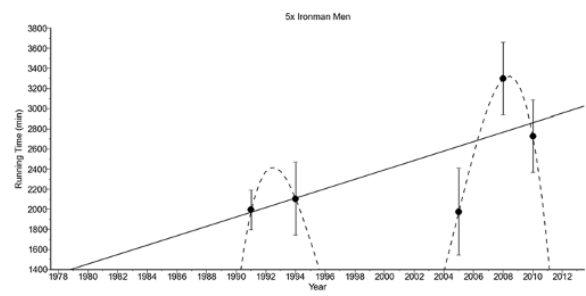

B

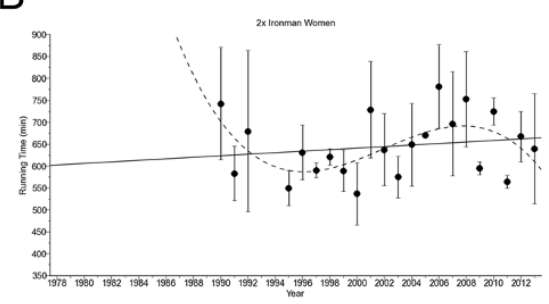

D

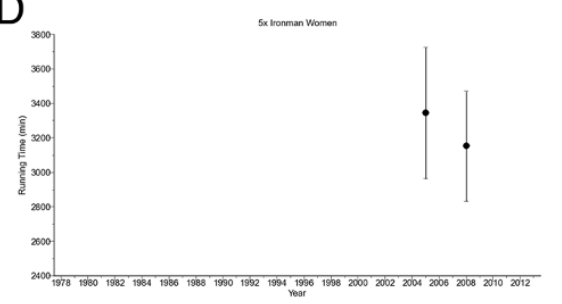

$\mathrm{F}$

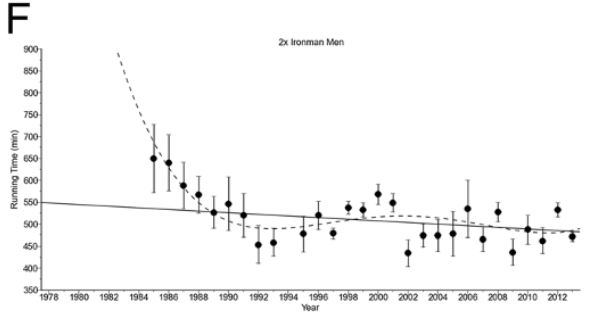

$\mathrm{H}$

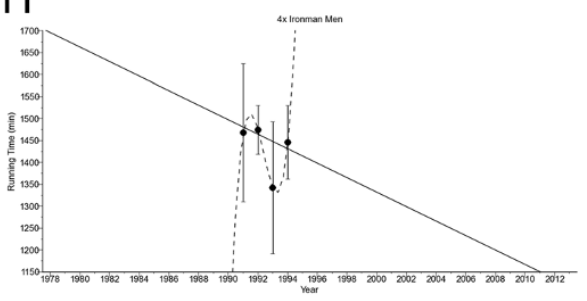

$J$

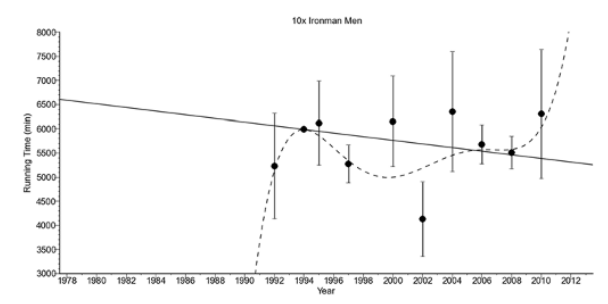

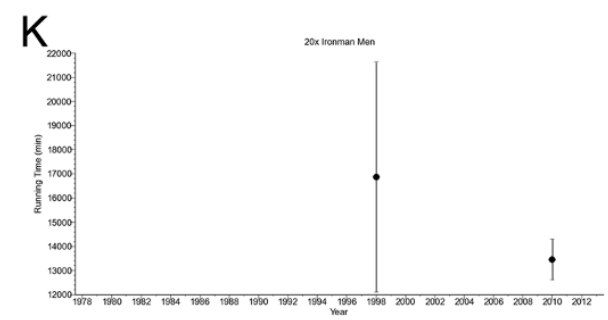

Figure $\mathbf{3}$ (See legend on next page.) 
(See figure on previous page.)

Figure 3 Running split times for the annual three fastest women and men from Ironman to Double Deca Iron ultra-triathlon for Ironman women (Panel A), Double Iron women (Panel B), Triple Iron women (Panel C), Quintuple Iron women (Panel D), Ironman men (Panel E), Double Iron men (Panel F), Triple Iron men (Panel G), Quadruple Iron men (Panel H), Quintuple Iron men (Panel I), Deca Iron men (Panel J), Double Deca Iron men (Panel K).

ultra-triathlon, however, race times increased linearly across years in women and non-linearly in men (i.e. polynomial regression 4th degree) (Table 5).

\section{Changes in sex difference across years}

The sex difference in the swimming split time (Figure 5) decreased non-linearly (Table 6) in 'Ironman Hawaii' (i.e. polynomial regression 5th degree). For the other distances, no changes were found (Table 6). In cycling (Figure 6), the sex difference decreased non-linearly (Table 7) in 'Ironman Hawaii' (i.e. polynomial regression 5th degree) but increased non-linearly in Triple Iron ultra-triathletes (i.e. polynomial regression 3rd degree) (Table 7). The sex difference in the running split time (Figure 7) decreased non-linearly (Table 8) in 'Ironman Hawaii' (i.e. polynomial regression 9th degree). In Triple Iron ultra-triathlon, the sex difference increased linearly (Table 8 ). For overall race time (Figure 8), the sex difference decreased non-linearly (Table 9) in 'Ironman Hawaii' (i.e. polynomial regression 8th degree). In Double Iron ultra-triathlon, the sex difference in overall race time increased non-linearly (i.e. polynomial regression 5th degree) (Table 9). In Triple Iron ultra-triathlon, the sex difference increased nonlinearly (i.e. polynomial regression 3rd degree) (Table 9).

Performance and sex difference of the fastest finishers ever When the fastest three women and men ever were considered (Figure 9), men were faster than women in both the split times (Panels A-C) and overall race times (Panel D). Figure 10 presents the sex difference for the three fastest finishers ever with split (Panels A-C) and overall race times (Panel D). Men were always faster than women. For the three fastest finishers ever, the sex differences in swimming were for Ironman, Double, Triple, Quadruple, Quintuple and Deca Iron ultra-triathlon $5.2 \pm$ $7.7 \%, 13.1 \pm 1.2 \%, 10.9 \pm 14.4 \%, 30.4 \pm 28.5 \%, 29.0 \pm 23.5 \%$, and $73.7 \pm 31.3 \%$, respectively. In cycling, the sex differences were $7.6 \pm 4.6 \%, 23.1 \pm 7.5 \%, 19.2 \pm 8.7 \%, 41.9 \pm$ $23.5 \%, 24.6 \pm 4.1 \%$, and $29.5 \pm 10.7 \%$, respectively. In running, the values were $9.5 \pm 3.9 \%, 27.1 \pm 5.3 \%, 20.1 \pm 5.1 \%$, $16.9 \pm 25.3 \%, 37.9 \pm 13.8 \%$, and $35.5 \pm 12.7 \%$, respectively. For overall race times, the sex differences were $8.5 \pm 2.5 \%$, $23.1 \pm 2.2 \%, \quad 18.4 \pm 1.2 \%, 29.3 \pm 19.0 \%, 30.0 \pm 11.0 \%$, and $34.6 \pm 4.6 \%$, respectively. The mean sex differences for swimming, cycling, running and overall race times for all distances from Ironman to Deca Iron ultra-triathlon were $27.0 \pm 17.8 \%$, $24.3 \pm 9.9 \%, 24.5 \pm 11.0 \%$, and $24.0 \pm 6.7 \%$, respectively. Figure 11 presents the relationship between sex difference and performance for split times (Panels A-C) and overall race times (Panel D) for the three fastest finishers ever. The sex difference increased with increasing race distance in swimming $\left(\mathrm{r}^{2}=0.93, P=0.001\right)$, but not in cycling $\left(r^{2}=0.18, P=0.38\right)$, running $\left(r^{2}=0.41, P=0.16\right)$, and overall race time $\left(r^{2}=0.59, P=0.07\right)$.

\section{Discussion}

This study investigated the sex difference in performance for each discipline and overall race time for the fastest competitors in ultra-distance triathlons from the Ironman distance in 'Ironman Hawaii' to the Double Deca

Table 4 Multi-level regression analyses for changes in running split times across years with correction for multiple finishes for the annual three fastest women and men

\begin{tabular}{|c|c|c|c|c|c|c|}
\hline Distance & Sex & $B$ & SE (B) & Stand. B & $\mathrm{T}$ & $P$ \\
\hline Ironman & Female & -1.228 & 0.115 & -0.734 & -10.647 & $<0.001$ \\
\hline Double Iron & Female & 1.744 & 1.768 & 0.122 & 0.986 & 0.328 \\
\hline Triple Iron & Female & 18.694 & 3.338 & 0.603 & 5.600 & $<0.001$ \\
\hline Quintuple Iron & Female & -64.111 & 95.465 & -0.318 & -0.672 & 0.539 \\
\hline Ironman & Male & -1.310 & 0.167 & -0.606 & -7.834 & $<0.001$ \\
\hline Double Iron & Male & -3.673 & 0.709 & -0.490 & -5.182 & $<0.001$ \\
\hline Triple Iron & Male & -0.261 & 1.600 & -0.019 & -0.163 & 0.871 \\
\hline Quadruple Iron & Male & -19.733 & 30.924 & -0.198 & -0.638 & 0.538 \\
\hline Quintuple Iron & Male & 46.406 & 17.218 & 0.599 & 2.695 & 0.018 \\
\hline Deca Iron & Male & 15.199 & 30.200 & 0.095 & 0.503 & 0.619 \\
\hline
\end{tabular}


Rüst et al. SpringerPlus 2014, 3:219

Page 9 of 17

http://www.springerplus.com/content/3/1/219
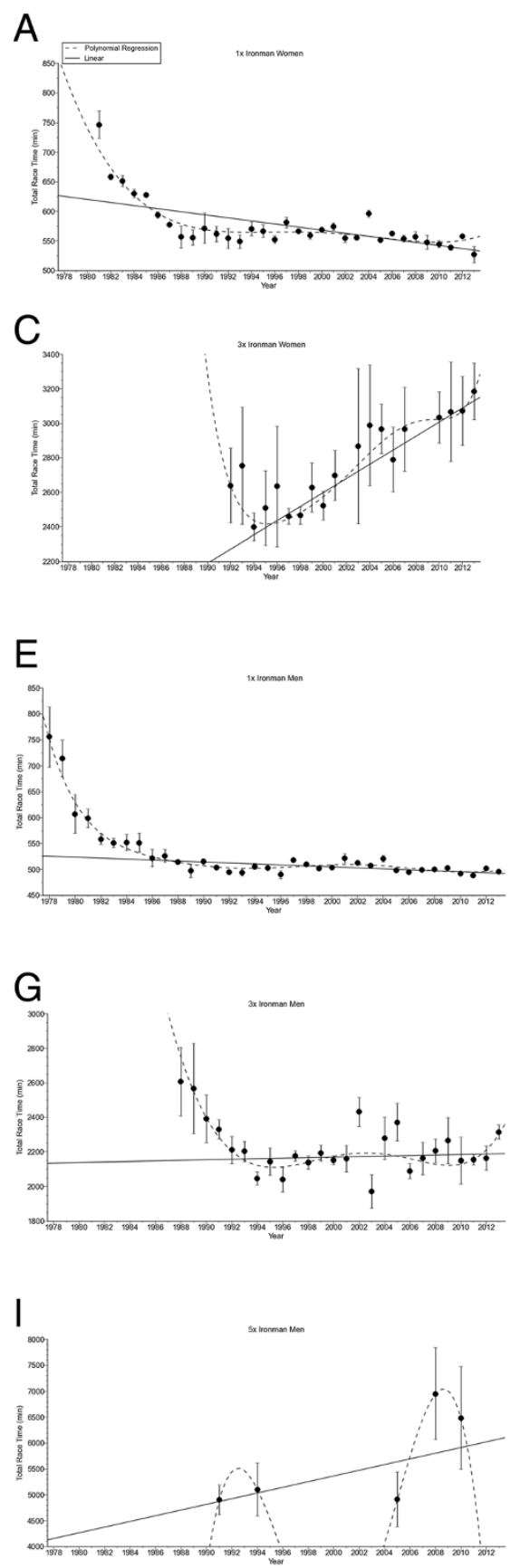

K
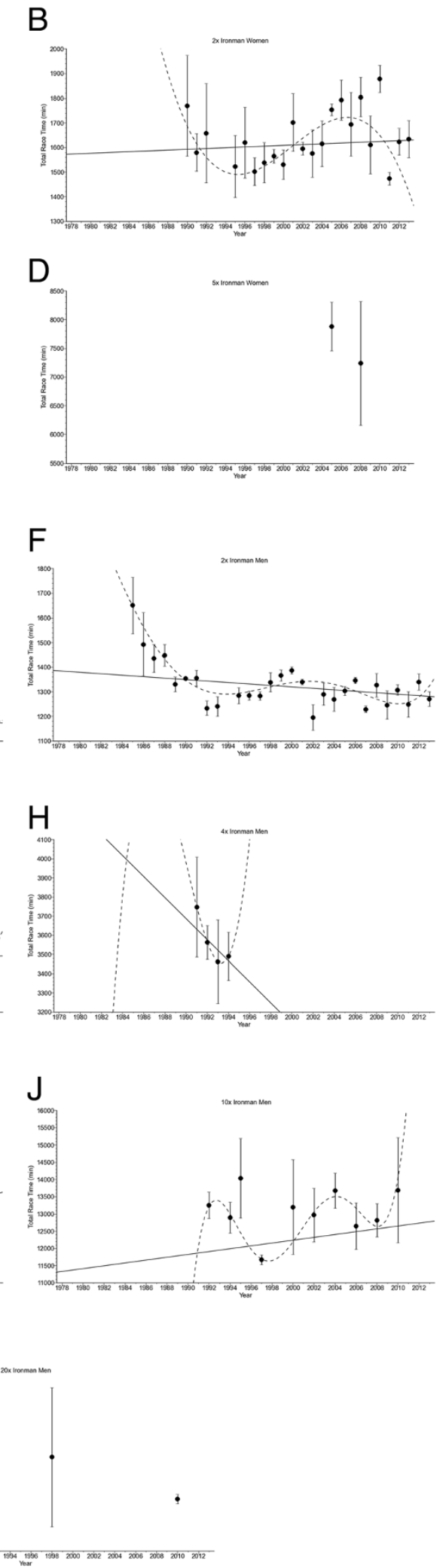

Figure 4 (See legend on next page.) 
(See figure on previous page.)

Figure 4 Overall race times for the annual three fastest women and men from Ironman to Double Deca Iron ultra-triathlon for Ironman women (Panel A), Double Iron women (Panel B), Triple Iron women (Panel C), Quintuple Iron women (Panel D), Ironman men (Panel E), Double Iron men (Panel F), Triple Iron men (Panel G), Quadruple Iron men (Panel H), Quintuple Iron men (Panel I), Deca Iron men (Panel J), Double Deca Iron men (Panel K).

Table 5 Multi-level regression analyses for change in overall race times cross years with correction for multiple finishes for the annual three fastest women and men

\begin{tabular}{|c|c|c|c|c|c|c|}
\hline Distance & Sex & $B$ & SE (ß) & Stand. B & $T$ & $P$ \\
\hline Ironman & Female & -3.031 & 0.345 & -0.665 & -8.781 & $<0.001$ \\
\hline Double Iron & Female & 3.404 & 2.436 & 0.172 & 1.398 & 0.167 \\
\hline Triple Iron & Female & 31.511 & 4.612 & 0.678 & 6.833 & $<0.001$ \\
\hline Quintuple Iron & Female & -214.111 & 223.258 & -0.432 & -0.959 & 0.392 \\
\hline Ironman & Male & -3.646 & 0.419 & -0.645 & -8.696 & $<0.001$ \\
\hline Double Iron & Male & -5.999 & 1.110 & -0.506 & -5.406 & $<0.001$ \\
\hline Triple Iron & Male & -6.678 & 2.479 & -0.295 & -2.694 & 0.009 \\
\hline Quadruple Iron & Male & -87.100 & 45.689 & -0.516 & -1.906 & 0.086 \\
\hline Quintuple Iron & Male & 84.992 & 29.276 & 0.627 & 2.903 & 0.012 \\
\hline Deca Iron & Male & 7.978 & 30.303 & 0.050 & 0.263 & 0.794 \\
\hline
\end{tabular}

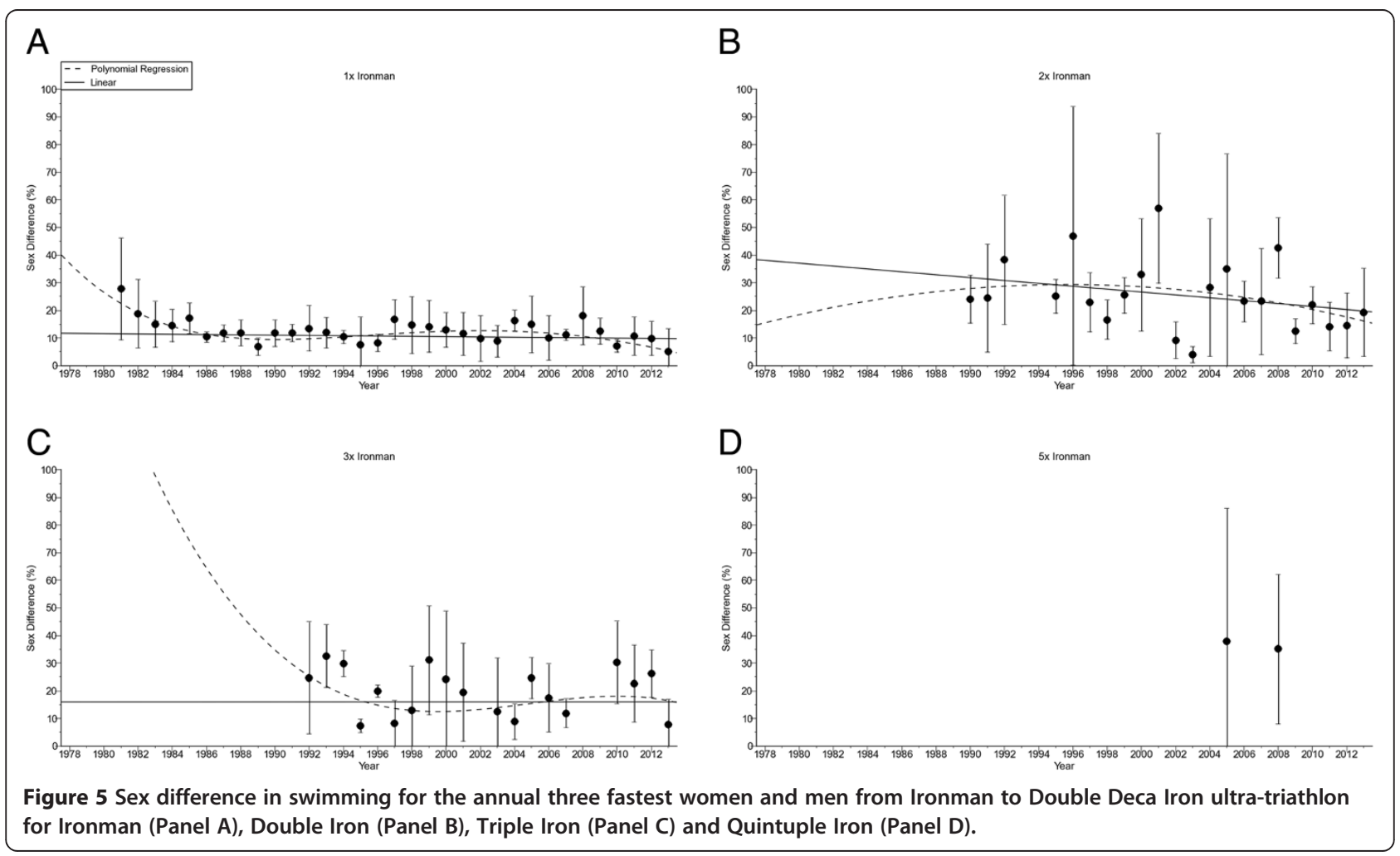


Table 6 Multi-level regression analyses for change in sex difference in swimming across years with correction for multiple finishes

\begin{tabular}{lccccc}
\hline Distance & $\boldsymbol{B}$ & $\mathbf{S E}(\boldsymbol{B})$ & Stand. $\boldsymbol{B}$ & $\mathbf{T}$ & $\boldsymbol{P}$ \\
\hline Ironman & -0.187 & 0.077 & -0.239 & -2.428 & 0.017 \\
Double Iron & -0.868 & 0.487 & -0.217 & -1.782 & 0.079 \\
Triple Iron & -0.169 & 0.285 & -0.080 & -0.593 & 0.556 \\
Quintuple Iron & -0.884 & 10.660 & -0.041 & -0.083 & 0.938 \\
\hline
\end{tabular}

Iron ultra-triathlon distance between 1978 and 2013. It was hypothesized that women would be able to reduce the gap with men in both swimming and running parts in ultra-triathlons. The main findings were that $(i)$ the fastest men were faster than the fastest women regarding overall race times, $(i i)$ women reduced the sex difference in the shorter ultra-triathlon distances (i.e. Ironman distance) but extended the sex difference in longer distances (i.e. Double and Triple Iron ultratriathlon) and (iii) in contrast to our hypothesis, the sex difference in the swimming split increased with increasing race distance.

Low female participation in ultra-endurance triathlon Female participation was highest in 'Ironman Hawaii' and lowest in Deca Iron ultra-triathlon. In Double Deca Iron ultra-triathlon, only one woman ever finished to date. The low number of women in the very long ultra-
Table 7 Multi-level regression analyses for change in sex difference in cycling across years with correction for multiple finishes

\begin{tabular}{lccccc}
\hline Distance & $\boldsymbol{B}$ & $\mathrm{SE}(\boldsymbol{\beta})$ & Stand. $\boldsymbol{B}$ & $\mathbf{T}$ & $\boldsymbol{P}$ \\
\hline Ironman & -0.155 & 0.050 & -0.299 & -3.089 & 0.003 \\
Double Iron & 0.277 & 0.214 & 0.160 & 1.294 & 0.200 \\
Triple Iron & 0.992 & 0.245 & 0.479 & 4.051 & $<0.001$ \\
Quintuple Iron & -16.983 & 3.264 & -0.933 & -5.204 & 0.007 \\
\hline
\end{tabular}

distance triathlons (i.e. longer than the Triple Iron ultratriathlon) might considerably influence the sex difference in triathlon performance. The offer of Ironman races is considerably higher (www.ironman.com/triathlon/ events) compared to races longer than the Ironman distance (www.iutasport.com). This large difference in offer might influence the participation. For running, Coast et al. (2004) compared the world best running performances at distances from $100 \mathrm{~m}$ to $200 \mathrm{~km}$ by the end of 2002. Men were faster than women with an average sex difference of $\sim 12.4 \%$ and longer distances were associated with greater sex differences. The authors argued that their results might be confounded by the lower number of women competing in longer distance events. Generally, men are over-represented in sports (Deaner 2013) and the faster performance in men compared to women is most probably due to men's greater training motivation (Deaner 2013). Popular modern
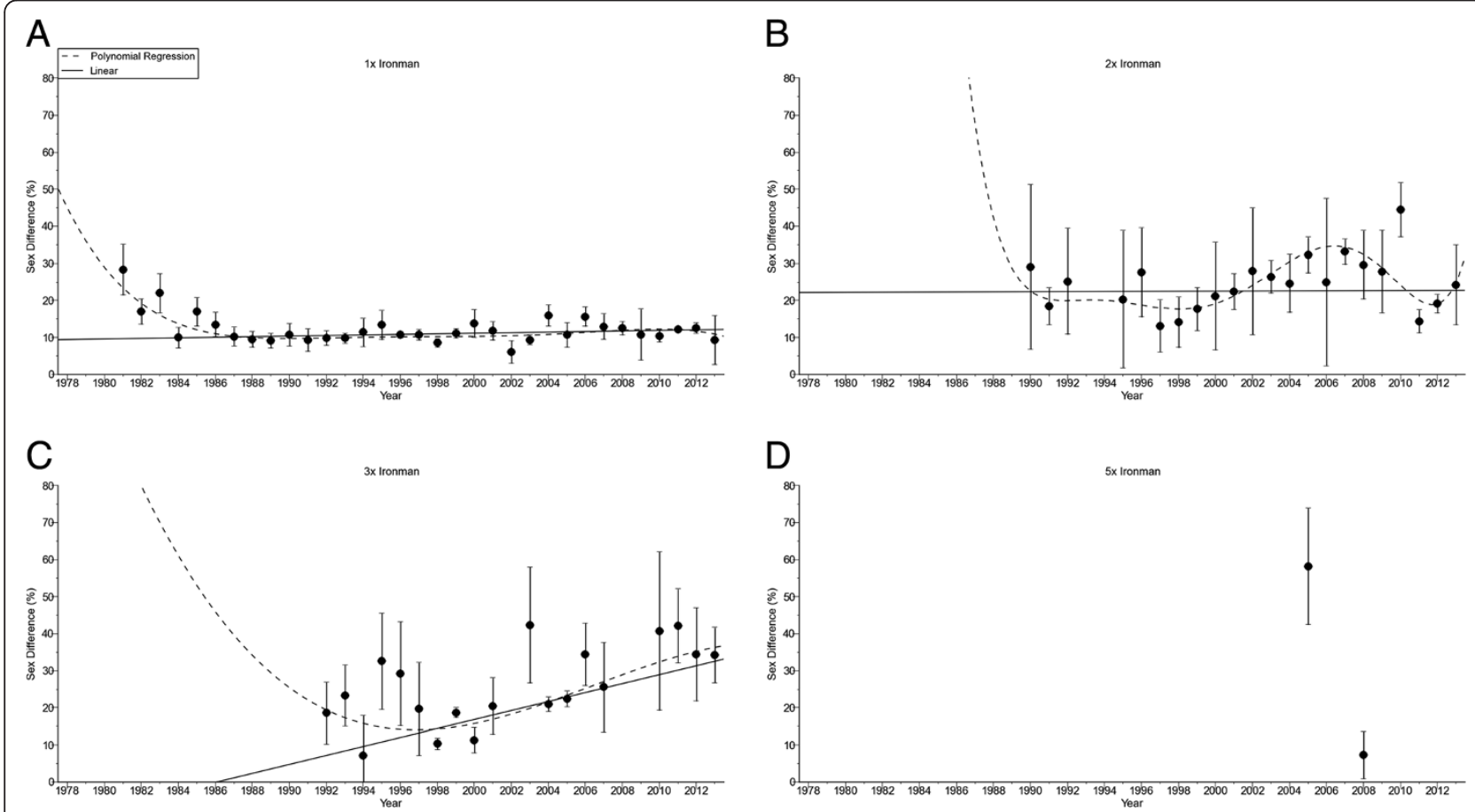

Figure 6 Sex difference in cycling for the annual three fastest women and men from Ironman to Double Deca Iron ultra-triathlon for Ironman (Panel A), Double Iron (Panel B), Triple Iron (Panel C) and Quintuple Iron (Panel D). 


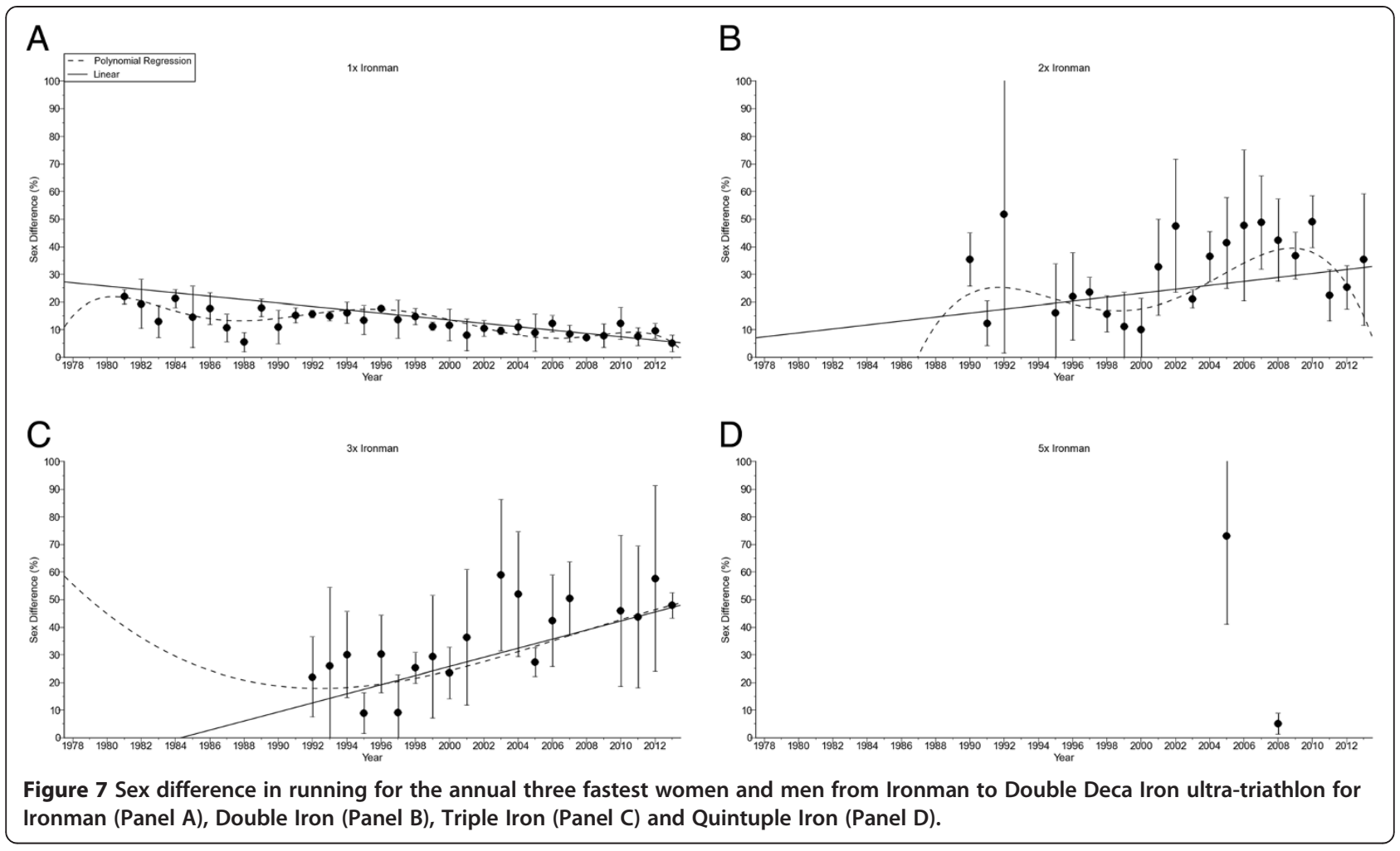

male sports require the skills needed for success in male-male physical competition (Lombardo 2012) whereas as female ultra-endurance athletes are task-oriented, internally motivated, health, and financially conscious individuals (Krouse et al. 2011).

\section{Differences in motivation between female and male athletes}

An explanation for the sex difference in both participation and performance in ultra-endurance could be a difference in the motivation between women and men to participate in an ultra-endurance race. Female participation in these ultra-triathlons was generally low at $\sim 12 \%$. Personality, motivation, and goal orientation have been investigated in endurance athletes such as runners (Frederick and Ryan 1993) and participants in different sports disciplines (Frederick and Ryan 1993; Gill and Overdorf 1994). Women were motivated to exercise

Table 8 Multi-level regression analyses for change in sex difference in running across years with correction for multiple finishes

\begin{tabular}{lccccc}
\hline Distance & $\boldsymbol{B}$ & $\mathrm{SE}(\boldsymbol{\beta})$ & Stand. $\boldsymbol{B}$ & $\mathbf{T}$ & $\boldsymbol{P}$ \\
\hline Ironman & -0.316 & 0.052 & -0.527 & -6.114 & $<0.001$ \\
Double Iron & 0.651 & 0.356 & 0.223 & 1.830 & 0.072 \\
Triple Iron & 1.668 & 0.385 & 0.504 & 4.332 & $<0.001$ \\
Quintuple Iron & -22.678 & 6.222 & -0.877 & -3.645 & 0.022 \\
\hline
\end{tabular}

regularly in order to reduce their body fat, to increase their physical fitness, or to improve their social interactions (Bond 2005; Frederick and Ryan 1993; Gill and Overdorf 1994; Hodge et al. 2008; Levy 2002). The aspect of competing and winning a race seemed to be of lower importance for women compared to men. For example, for female marathoners, the aspect of social affiliation and improving physical fitness is more important than achievement and personal accomplishment (Masters and Ogles 1995; Ogles and Masters 2003). Krouse et al. (2011) investigated the influence of motivation, goal orientation and training on performance for female ultra-marathoners. General health orientation and psychological coping were the strongest motivational factors for female ultra-marathoners.

\section{Sex difference and race distance}

It was hypothesized that women would reduce the gap with men in both swimming and running parts of ultratriathlons. Although women reduced the sex difference across years in the shorter ultra-triathlon distances such as the Ironman distance, they extended the sex difference in longer distances such as the Double and the Triple Iron ultra-triathlon distance. Furthermore, the sex difference remained unchanged at $\sim 24-27 \%$ for split and overall race times when the fastest three performances ever for all distances from Ironman to Double Deca Iron ultra-triathlon were considered. Recent 


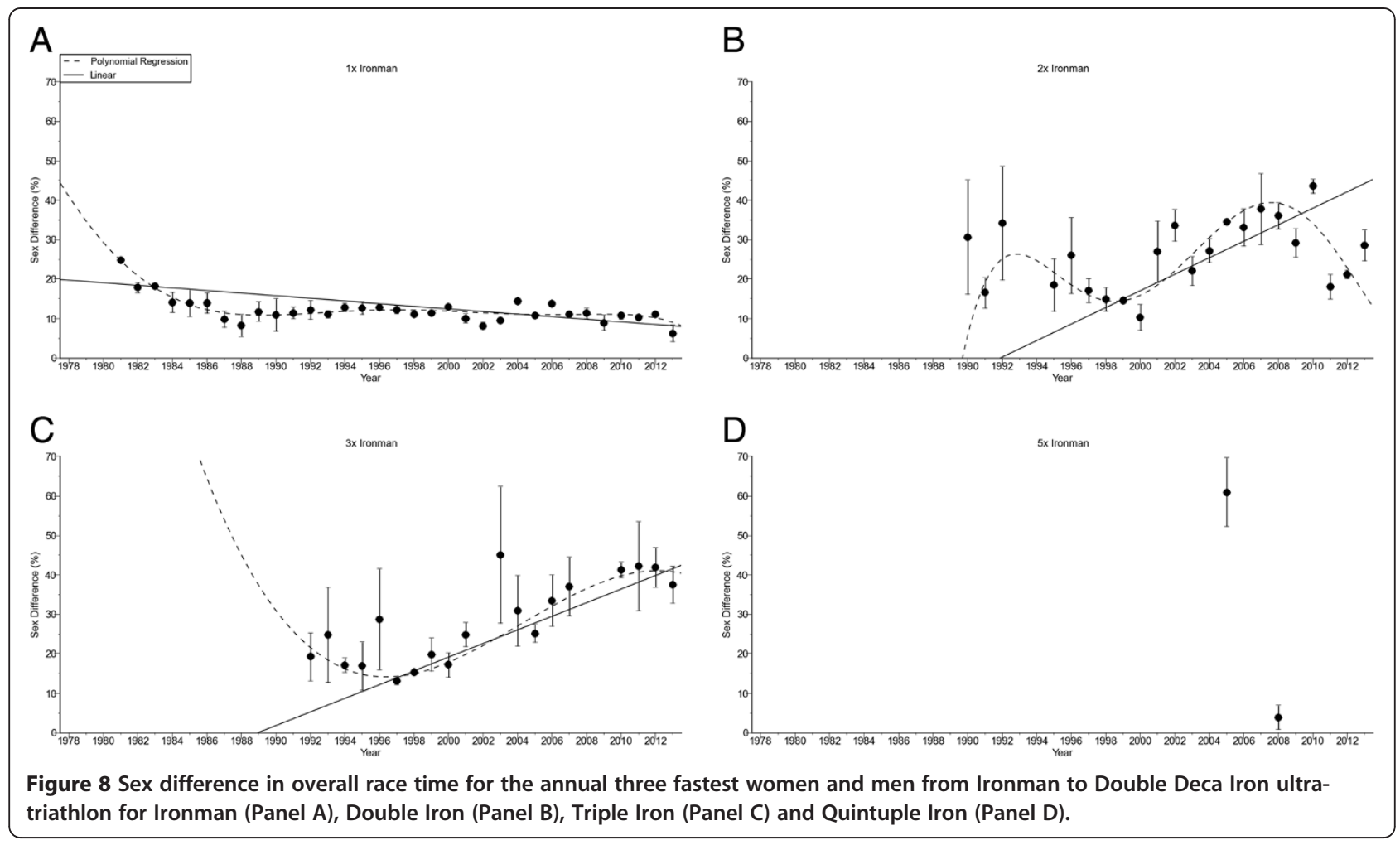

studies investigating ultra-endurance athletes competing in single disciplines showed that women reduced the gap to men in ultra-swimming (Rüst et al. 2014) and ultra-running (Zingg et al. 2014b). Although women were able to outperform men in ultra-swimming under certain conditions (i.e. very long distance and very cold water) (Knechtle et al. 2014), is seemed unlikely that women will outperform men in ultra-marathon running (Zingg et al. 2014b) and ultra-cycling (Rüst et al. 2013).

The sex difference in performance between female and male endurance athletes might be explained in part by differences in anthropometric characteristics such as skeletal muscle mass and body fat between female and male athletes. Knechtle et al. (2011a) argued that the increase in sex difference with increasing length of an ultra-endurance performance such as an ultra-triathlon was most probably due to the lower skeletal muscle mass in women compared to men. It has been shown

Table 9 Multi-level regression analyses for change in sex difference in overall race time across years with correction for multiple finishes

\begin{tabular}{lccccc}
\hline Distance & $\boldsymbol{B}$ & $\mathbf{S E}(\boldsymbol{\beta})$ & Stand. $\boldsymbol{\beta}$ & $\mathbf{T}$ & $\boldsymbol{P}$ \\
\hline Ironman & -0.200 & 0.032 & -0.538 & -6.279 & $<0.001$ \\
Double Iron & 0.411 & 0.179 & 0.276 & 2.295 & 0.025 \\
Triple Iron & 1.254 & 0.181 & 0.683 & 6.943 & $<0.001$ \\
Quintuple Iron & -19.022 & 1.769 & -0.983 & -10.755 & $<0.001$ \\
\hline
\end{tabular}

that male ultra-endurance athletes had a higher skeletal muscle mass than female ultra-endurance athletes (Knechtle et al. 2010a; 2010b; 2011b; Weitkunat et al. 2012). Male Ironman triathletes with $\sim 41 \mathrm{~kg}$ skeletal muscle mass had a $\sim 46 \%$ higher skeletal muscle mass compared to female Ironman triathletes with $\sim 28 \mathrm{~kg}$ of skeletal muscle mass (Knechtle et al. 2010a). For ultrarunners, male ultra-runners with $\sim 38 \mathrm{~kg}$ skeletal muscle mass (Knechtle et al. 2011b) had a 38\% higher muscle mass compared to female ultra-runners with $\sim 27.4 \mathrm{~kg}$ (Knechtle et al. 2010b). For ultra-swimmers, the sex difference in skeletal muscle mass was considerably higher compared to runners (Weitkunat et al. 2012). Male open-water ultra-swimmers with $\sim 42 \mathrm{~kg}$ skeletal muscle mass had a $\sim 45 \%$ more skeletal muscle mass compared to female open-water ultra-swimmers with $\sim 29 \mathrm{~kg}$ skeletal muscle mass (Weitkunat et al. 2012). These differences in skeletal muscle mass between female and male ultra-endurance athletes might explain the increase in sex difference with increasing length of an endurance performance.

Another major predictor variable for a successful outcome in endurance competitions is percent body fat. It has been shown that lower body fat was associated with faster race times in men (Knechtle et al. 2011c; Rüst et al. 2012). Mean body fat percentages for male and female Ironman triathletes were $\sim 14 \%$ and $\sim 23 \%$, respectively (Knechtle et al. 2010a). The higher skeletal 


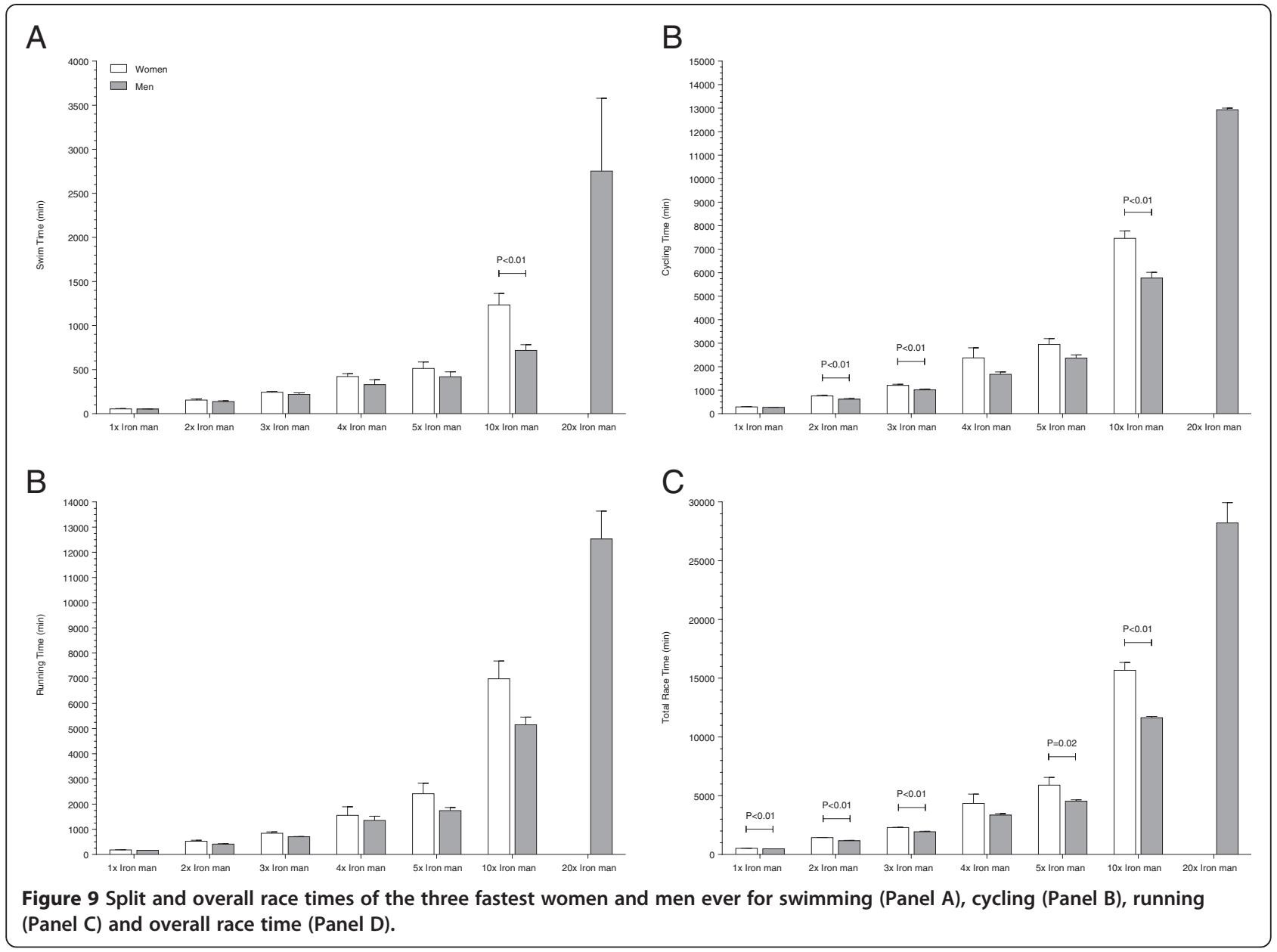

muscle mass and the lower body fat percentage in men may support the theory of a biological based performance difference of female and male ultra-endurance athletes.

A further aspect is that performance in cycling is related to leg power (Hopker et al. 2010). An ultra-endurance performance leads to a decrease in lean body mass (Schütz et al. 2013). Since women have a lower skeletal muscle mass, a prolonged cycling performance might lead to a higher relative decrease in lower leg skeletal muscle mass in women compared to men and therefore, cycling performance becomes more reduced in women compared to men.

\section{Women extended the sex difference in swimming with increasing race distance}

It was hypothesized that women would be able to achieve better swimming performances than men but in contrast to our hypothesis, the sex difference in the swimming split increased with increasing race distance in these ultra-triathletes. A potential explanation could be the 'history' of the athletes. Triathletes have to compete in three different disciplines and the background might be different for each athlete. Since cycling and running performance are predictive for ultra-triathlon performance (Knechtle et al. 2007; Knechtle and Kohler 2009), the background of an ultra-triathlete might rather be running or cycling than swimming. For elite openwater ultra-swimmers, however, women improved in long-distance swimming in recent years (Eichenberger et al. 2012b; Vogt et al. 2013; Zingg et al. 2014a). The sex difference in the world's best $10 \mathrm{~km}$ open-water swimmers is only $\sim 7 \%$ (Vogt et al. 2013). In open-water swimming, women are even able to achieve men's performance (Eichenberger et al. 2012b). However, when the the changes in swimming speeds and sex differences for elite male and female swimmers competing in $5 \mathrm{~km}$, $10 \mathrm{~km}$ and $25 \mathrm{~km}$ open-water FINA World Cup races held between 2000 and 2012 were investigated (Zingg et al. 2014c), elite female open-water ultra-distance swimmers improved in $10 \mathrm{~km}$ but impaired in $25 \mathrm{~km}$ leading to a linear decrease in sex difference in $10 \mathrm{~km}$ and a linear increase in sex difference in $25 \mathrm{~km}$. The linear changes in sex differences suggested that women would improve in the near future in $10 \mathrm{~km}$, but not in $25 \mathrm{~km}$ (Zingg et al. 2014c). 


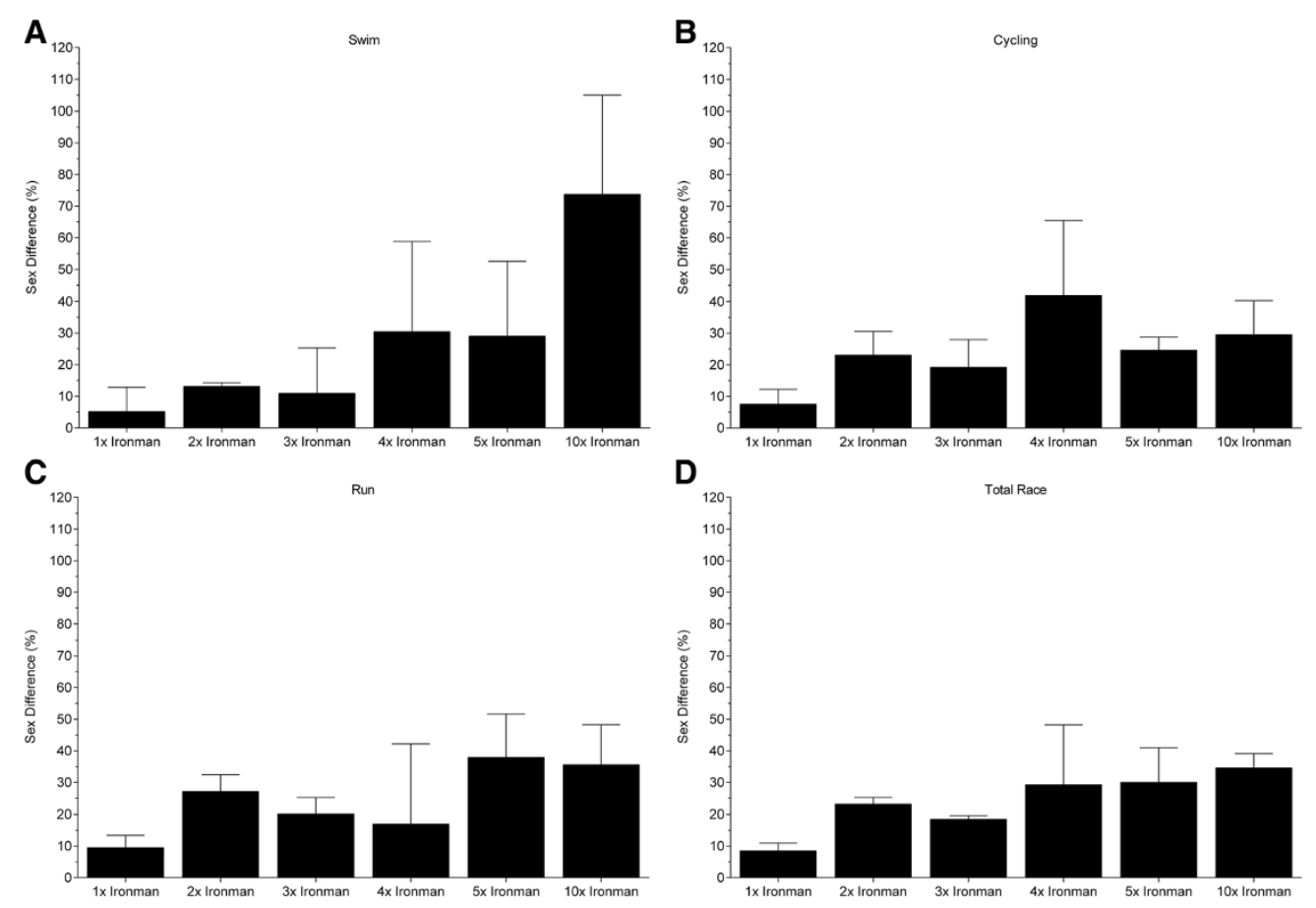

Figure 10 Sex difference in split and overall race times of the three fastest women and men ever for swimming (Panel A), cycling (Panel B), running (Panel C) and overall race time (Panel D).
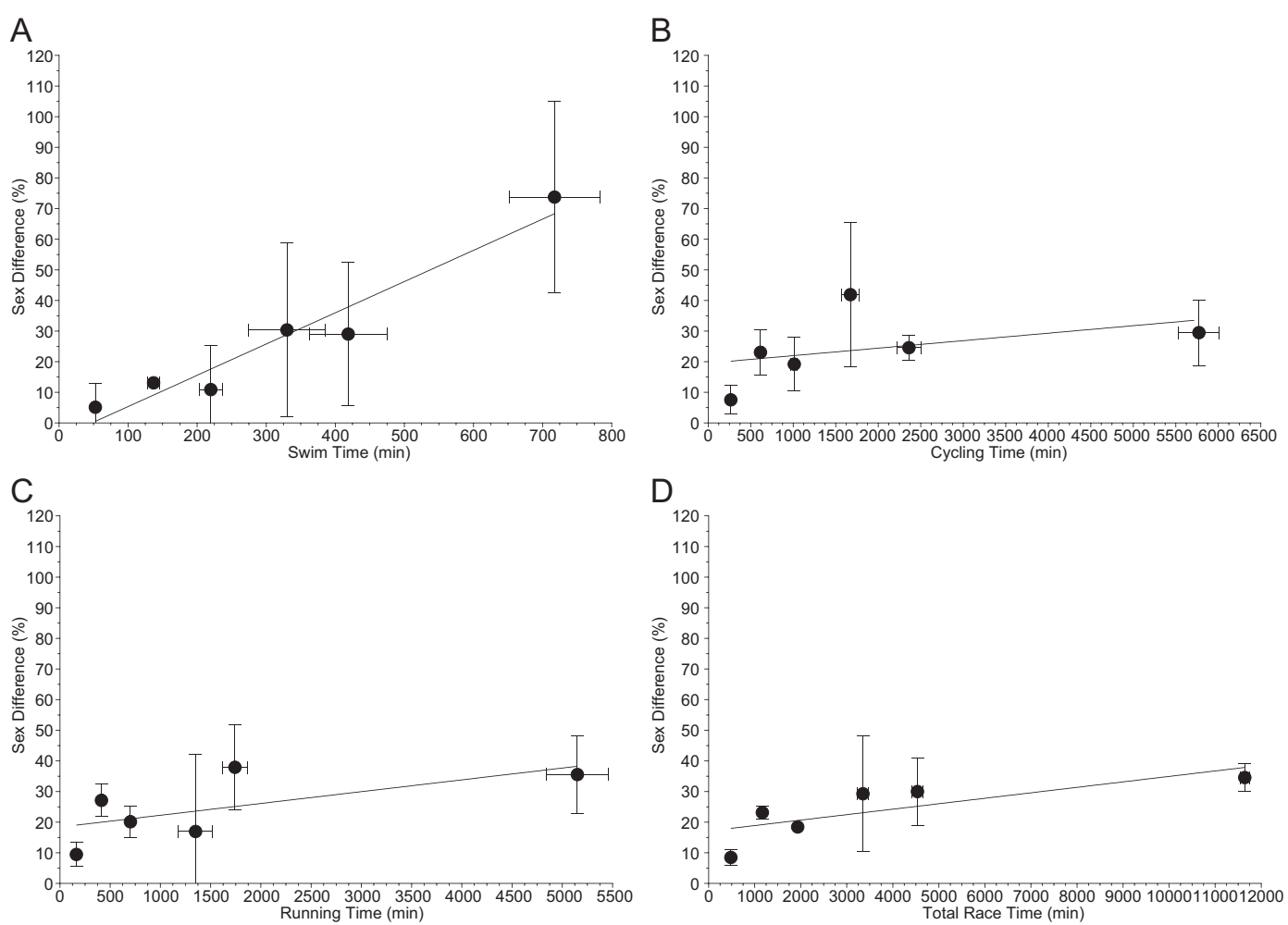

Figure 11 Sex difference in relation to split and overall race times for the three fastest competitors ever across all distances for swimming (Panel A), cycling (Panel B), running (Panel C) and overall race time (Panel D). 


\section{Conclusions}

Although women reduced the sex difference in the shorter ultra-triathlon distances (i.e. Ironman distance), the sex difference in longer triathlon distances (i.e. Double and the Triple Iron ultra-triathlon distance) remained greater compared to the single Ironman distance. The sex difference for split times and overall race times remained unchanged at $\sim 24-27 \%$ for the fastest three performances ever for all distances. Additionally, the sex difference in the swimming split increased significantly with increasing race distance. The most likely reasons for the sex difference in ultra-triathlon performance are the lower number in female participation in these races, the lower muscle mass in women compared to men and the different motivational trends in women compared to men. It seems very unlikely that women will ever outrun men in ultra-distance triathlons from the Ironman to the Double Deca Iron ultra-triathlon distance.

\section{Competing interests}

The authors declare that they have no competing interests.

\section{Authors' contributions}

CR drafted the manuscript and performed the statistical analyses, TR helped in drafting the manuscript, BK collected the data and helped in drafting the manuscript. All authors read and approved the final manuscript.

\section{Acknowledgements}

The authors would like to thank the race directors for providing us their race results.

\section{Author details}

${ }^{1}$ Institute of General Practice and Health Services Research, University of Zurich, Zurich, Switzerland. ${ }^{2}$ Gesundheitszentrum St. Gallen, Vadianstrasse 26, St. Gallen 9001, Switzerland.

\section{Received: 13 January 2014 Accepted: 22 April 2014}

Published: 1 May 2014

\section{References}

Badwater, website www.badwater.com/results/index.html

Beneke R, Leithäuser RM, Doppelmayr M (2005) Women will do it in the long run. Br J Sports Med 39:410

Bond K (2005) Running for their lives: A qualitative analysis of the exercise experience of female recreational runners. Women Sport Phys Activity J 14:69-82

Coast JR, Blevins JS, Wilson BA (2004) Do gender differences in running performance disappear with distance? Can J Appl Physiol 29:139-145

da Fonseca-Engelhardt K, Knechtle B, Rüst CA, Knechtle P, Lepers R, Rosemann T (2013) Participation and performance trends in ultra-endurance running races under extreme conditions - 'Spartathlon' versus 'Badwater'. Extrem Physiol Med 2:15

Deaner RO (2013) Distance running as an ideal domain for showing a sex difference in competitiveness. Arch Sex Behav 42:413-428

Eichenberger E, Knechtle B, Rüst CA, Knechtle P, Lepers R, Rosemann T (2012a) No gender difference in peak performance in ultra-endurance swimming performance - analysis of the 'Zurich 12-h Swim' from 1996 to 2010. Chin J Physiol 55:346-351

Eichenberger E, Knechtle B, Knechtle P, Rüst CA, Rosemann T, Lepers R (2012b) Best performances by men and women open-water swimmers during the 'English Channel Swim' from 1900 to 2010. J Sports Sci 30:1295-1301

Frederick C, Ryan R (1993) Differences in motivation for sport and exercise and their relations with participation and mental health. J Sport Behavior 16:124-146

Gill K, Overdorf V (1994) Incentives for exercise in younger and older women. J Sport Behav 17:87-97
Hodge K, Allen J, Smellie L (2008) Motivation in masters sports: Achievement and social goals. Psychol Sport Exerc 9:157-176

Hoffman MD (2008) Ultramarathon trail running comparison of performance-matched men and women. Med Sci Sports Exerc 40:1681-1686

Hopker J, Jobson S, Carter H, Passfield L (2010) Cycling efficiency in trained male and female competitive cyclists. J Sports Sci Med 9:332-337

International Ultra-Triathlon Association (IUTA), website http://eu.ironman.com/ events/triathlon-races.aspx\#axzz3114PEJra

Ironman events (Ironman), website http://eu.ironman.com/events/triathlon-races. aspx\#axzz3114PEJra

Ironman World Championship, website www.ironmanworldchampionship.com

Knechtle B, Kohler G (2009) Running performance, not anthropometric factors, is associated with race success in a Triple Iron Triathlon. Br J Sports Med 43:437-441

Knechtle B, Duff B, Amtmann G, Kohler G (2007) Cycling and running performance, not anthropometric factors, are associated with race performance in a Triple Iron Triathlon. Res Sports Med 15:257-269

Knechtle B, Duff B, Schulze I, Kohler G (2008) The effects of running 1,200 km within 17 days on body composition in a female ultrarunner-Deutschlandlauf 2007. Res Sports Med 16:167-188

Knechtle B, Wirth A, Baumann B, Knechtle P, Rosemann T, Senn O (2010a) Differential correlations between anthropometry, training volume, and performance in male and female Ironman triathletes. J Strength Cond Res 24:2785-2793

Knechtle B, Senn O, Imoberdorf R, Joleska I, Wirth A, Knechtle P, Rosemann T (2010b) Maintained total body water content and serum sodium concentrations despite body mass loss in female ultra-runners drinking ad libitum during a $100 \mathrm{~km}$ race. Asia Pac J Clin Nutr 19:83-90

Knechtle B, Knechtle P, Lepers R (2011a) Participation and performance trends in ultra-triathlons from 1985 to 2009. Scand J Med Sci Sports 21:e82-e90

Knechtle B, Senn O, Imoberdorf R, Joleska I, Wirth A, Knechtle P, Rosemann T (2011b) No fluid overload in male ultra-runners during a $100 \mathrm{~km}$ ultra-run. Res Sports Med 19:14-27

Knechtle B, Knechtle P, Rüst CA, Rosemann T (2011c) A comparison of anthropometric and training characteristics of Ironman triathletes and Triple Iron ultra-triathletes. J Sports Sci 29:1373-1380

Knechtle B, Rosemann T, Lepers R, Rüst CA (2014) Women outperform men in ultra-distance swimming - the 'Manhattan Island Marathon Swim' from 1983 to 2013. Int J Sports Physiol Perform, 2014 Feb 28. [Epub ahead of print]

Krouse RZ, Ransdell LB, Lucas SM, Pritchard ME (2011) Motivation, goal orientation and training habits of women ultrarunners. J Strength Cond Res 25:2835-2842

Lenherr R, Knechtle B, Rüst CA, Rosemann T, Lepers R (2012) From Double Iron to Double Deca Iron ultra-triathlon - a retrospective data analysis from 1985 to 2011. Phys Cultur Sport Stud Res 54:55-67

Lepers R (2008) Analysis of Hawaii ironman performances in elite triathletes from 1981 to 2007. Med Sci Sports Exerc 40:1828-1834

Lepers R, Maffiuletti NA (2011) Age and gender interactions in ultraendurance performance: insight from the triathlon. Med Sci Sports Exerc 43:134-139

Levy S (2002) Women and the personal meaning of competition: a qualitative investigation. Women Sport Phys Activity J 11:107-119

Lombardo MP (2012) On the evolution of sport. Evol Psychol 10:1-28

Masters K, Ogles B (1995) An investigation of the different motivations of marathon runners with varying degrees of experience. J Sport Behav 18:69-79

McLean SP, Hinrichs RN (1998) Sex differences in the centre of buoyancy location of competitive swimmers. J Sports Sci 16:373-383

Ogles B, Masters K (2003) A typology of marathon runners based on cluster analysis of motivation. J Sport Behav 26:69-85

Perreault L, Lavely JM, Kittelson JM, Horton TJ (2004) Gender differences in lipoprotein lipase activity after acute exercise. Obes Res 12:241-249

Reinboud W (2004) Linear models can't keep up with sport gender gap. Nature 432(7014):147

Riddell MC, Partington SL, Stupka N, Armstrong D, Rennie C, Tarnopolsky MA (2003) Substrate utilization during exercise performed with and without glucose ingestion in female and male endurance trained athletes. Int I Sport Nutr Exerc Metab 13:407-421

Roepstorff C, Steffensen CH, Madsen M, Stallknecht B, Kanstrup IL, Richter EA, Kiens B (2002) Gender differences in substrate utilization during submaximal exercise in endurance-trained subjects. Am J Physiol Endocrinol Metab 282:E435-E447 
Rüst CA, Knechtle B, Knechtle P, Rosemann T (2012) Similarities and differences in anthropometry and training between recreational male 100-km ultra-marathoners and marathoners. J Sports Sci 30:1249-1257

Rüst CA, Knechtle B, Rosemann T, Lepers R (2013) Men cross America faster than women-the "Race Across America" from 1982 to 2012. Int J Sports Physiol Perform 8:611-617

Rüst CA, Lepers R, Rosemann T, Knechtle B (2014) Will women soon outperform men in open-water ultra-distance swimming in the 'Maratona del Golfo Capri-Napoli'? Springerplus 3:86

Schumacher YO, Mueller P, Keul J (2001) Development of peak performance in track cycling. J Sports Med Phys Fitness 41:139-146

Schütz UH, Billich C, König K, Würslin C, Wiedelbach H, Brambs HJ, Machann J (2013) Characteristics, changes and influence of body composition during a $4486 \mathrm{~km}$ transcontinental ultramarathon: results from the Transeurope Footrace mobile whole body MRI-project. BMC Med 11:122

Shoak MA, Knechtle B, Knechtle P, Rüst CA, Rosemann T, Lepers R (2013) Participation and performance trends in ultracycling. Open Access J Sports Med 4:41-51

Tarnopolsky L, MacDougall JD, Atkinson SA, Tarnopolsky MA, Sutton JR (1990) Gender differences in substrate for endurance exercise. J Appl Physiol 68:302-308

Vogt P, Rüst CA, Rosemann T, Lepers R, Knechtle B (2013) Analysis of 10 km swimming performance of elite male and female open-water swimmers. Springerplus 2:603

Weitkunat T, Knechtle B, Knechtle P, Rüst CA, Rosemann T (2012) Body composition and hydration status changes in male and female open-water swimmers during an ultra-endurance event. J Sports Sci 30:1003-1013

Zingg M, Knechtle B, Rüst CA, Rosemann T, Lepers R (2013) Age and gender difference in non-drafting ultra-endurance cycling performance - the 'Swiss Cycling Marathon'. Extreme Physiol Med 2:18

Zingg MA, Rüst CA, Rosemann T, Lepers R, Knechtle B (2014a) Analysis of swimming performance in FINA World Cup long-distance open water races. Extreme Physiol Med 3:2

Zingg MA, Karner-Rezek K, Rosemann T, Knechtle B, Lepers R, Rüst CA (2014b) Will women outrun men in ultra-marathon road races from $50 \mathrm{~km}$ to 1,000 $\mathrm{km}$ ? Springerplus 3:97

Zingg MA, Rüst CA, Rosemann T, Lepers R, Knechtle B (2014C) Analysis of sex differences in open-water ultra-distance swimming performances in the FINA World Cup races in $5 \mathrm{~km}, 10 \mathrm{~km}$ and $25 \mathrm{~km}$ from 2000 to 2012. BMC Sports Sci Med Rehabil 6:7

doi:10.1186/2193-1801-3-219

Cite this article as: Rüst et al:: Performance and sex difference in ultra-triathlon performance from Ironman to Double Deca Iron ultra-triathlon between 1978 and 2013. SpringerPlus 2014 3:219.

\section{Submit your manuscript to a SpringerOpen ${ }^{\odot}$ journal and benefit from:}

- Convenient online submission

- Rigorous peer review

- Immediate publication on acceptance

- Open access: articles freely available online

- High visibility within the field

- Retaining the copyright to your article

Submit your next manuscript at springeropen.com 